\title{
Otros procedimientos de intervención en la ciudad contemporánea: sobre la re-densificación
}

\author{
Fernando Bajo Martínez de Murguía ${ }^{1}$
}

Recibido: 08-07-2020 | en su versión final: 09-11-2020

Resumen

\begin{abstract}
Un proceso de regeneración urbana tan actual y discutido como es el de la re-densificación, puede convertirse en origen de conflictos si no se desarrolla con conocimiento y de acuerdo con un procedimiento contrastado. Para ello es importante analizar sus fundamentos, con el fin de adelantarse a los posibles problemas derivados, y aplicar una metodología adaptada para cada caso, pero que de forma general obedezca a una serie de fases reconocibles. Son los casos prácticos, es decir la experiencia, la que ilumina la aplicación de esta figura urbanística plena de sentido en el momento en el que nos encontramos. Pero que precisamente por su trascendencia y capacidad transformadora del ámbito urbano puede convertirse en factor controvertido; incluso susceptible de recelo tanto desde el poder político como de la ciudadanía. Conviene aclarar ciertos aspectos de la re-densificación con el fin de conocer su utilidad para el desarrollo de una práctica del diseño y planeamiento urbano sensatos. Flexible y respetuosa no solo con el programa de necesidades inicial, sino también con la identidad propia del ámbito en el que se introduce. Sin olvidar su naturaleza acorde con el paradigma de una realidad como la urbana, que nunca termina de agotar sus potencialidades. Reinventándose sin necesidad de incrementar la demanda de recursos externos. Para ello, se analiza un caso real; aportación original en la que se investigan sus derivas a la vez que se sistematiza el proceso seguido para su desarrollo. Con el objetivo final de poder incluir posibles mejoras en situaciones replicables.
\end{abstract}

Palabras clave: densificación; compacidad legible, sostenibilidad urbana; procedimientos urbanos

Citación

Bajo Martínez de Murguía, F. (2021). Otros procedimientos de intervención en la ciudad contemporánea: sobre la re-densificación. ACE: Architecture, City and Environment, 15(45), 9527. DOI: http://dx.doi.org/10.5821/ace.15.45.9527

\section{Another Kind of Procedures for Today's City Planning and Design: About Re-densification}

Abstract

Such a contemporary and debated urban regeneration process as the one so-called re-densification, can easily lead to a storm of social and political conflicts. At least unless it is not carried out wisely, and according to a proper-balanced program. In fact, it is especially important to analyze its fundamentals, in order to preview possible out coming troubles. Using a refined and accurate methodology focused on every single case study and adapted to a sequence of well-known phases. It is the case study; let's mean the experience, what must lead the practice under this urban procedure that makes absolute sense in today's context. However, because of its power of transformation within the urban environment, it can also become a source of controversy and, in addition, the target of opposed political interests. Therefore, it is important to make this concept clear in order to know about its use in every day and common-sense urbanism. Flexible and sensitive not only to the needs of every part of the city, but with their identity too. Not forgetting its coherency with the paradigm that applies to our real cities in terms of sustainability. Reinventing them without the increasing demand of external resources. To do this, a real case is analyzed in which its drifts are investigated while the process followed for its development is systematized, with the final objective of being able to include possible improvements in replicable situations.

Keywords: density increase; vivid compacity; urban sustainability; urban procedures

1 Dr. Arquitecto, Profesor Titular, Departamento de Arquitectura, Escuela Superior de Arquitectura de Donostia-San Sebastián, Universidad del País Vasco, UPV-EHU (ORCID: 0000-0001-8945-3433). Correo de contacto: fernando.bajo@ehu.es

ACE, X (XX) CC BY-ND 3.0 ES | UPC Barcelona, España | Otros procedimientos de intervención en la ciudad 


\section{Introducción}

El presente artículo tiene como objetivo profundizar en el conocimiento de una novedosa herramienta de intervención urbana aplicada en dos ámbitos de suelo urbano recientemente consolidados. Se trata del proceso de re-densificación como incremento de la edificabilidad física, aplicado a los nuevos sectores de desarrollo tanto al Este como al Oeste de la ciudad de Vitoria-Gasteiz, capital del País Vasco. Una revisión de planeamiento general (tal y como su denominación técnica indica), que tanto por sus características como por su dimensión presenta un gran interés como caso de estudio. No adscrita en exclusiva a la regeneración urbana, sino más bien a la búsqueda iterativa de una densidad adecuada para partes muy determinadas de la ciudad. Y un reflejo de los conflictos generados entre la administración tutelar del proceso y los vecinos afectados: como un ejemplo más del que nunca llueve a gusto de todos. Del mismo modo extrae lecciones prácticas de la aplicación de un tipo de intervención urbana muy particular como es el de la re-densificación, una práctica sin duda eficaz y novedosa en nuestro país (López de Munain, 2012), a la hora de corregir intensidades de usos, discontinuidades, o incluso renovaciones urbanas puntuales de importancia. Pero más compleja de lo que en principio pudiera parecer, y no exenta de problemas en su aplicación (Gaja i Díaz, 2005).

Puesto que se trata de una medida práctica ajustable a las necesidades reales de cada caso, la redensificación urbana no demanda condiciones específicas de partida (por lo menos no otras que la voluntad política soportada en el consenso ciudadano). Y en esto tienen mucho que decir los nuevos desarrollos urbanos hacia el Este y Oeste de Vitoria-Gasteiz, denominados como Salburua y Zabalgana respectivamente, que hasta cierto punto y gracias a esta herramienta, han corregido el excesivo consumo de suelo urbanizable de la ciudad. Un hecho significativo que, entre otros, le permitió convertirse en European Green Capital durante el año 2012.

Hemos de ser conscientes de que este escrito, con suficiente perspectiva, supone una recapitulación sobre el planteamiento inicial que describe los primeros pasos de un proceso real, además de criticar y poner en orden una serie de aspectos procedimentales sufridos durante el desarrollo de un caso particular de la práctica urbanística actual. Uno de los que, por su carácter novedoso, magnitud y trascendencia mediática, merece la pena revisitar con cierto detalle, puntualizando algunos matices. Porque además no es ajeno a ciertos conflictos impredecibles desde un principio.

La idea de fondo o hipótesis defendida es que este instrumento de intervención urbana puede ser un complemento ideal para el planeamiento de rango general, incrementando la cultura del Plan respecto la cultura del Proyecto (López de Lucio, 1993). O en su caso incluso una alternativa más eficaz que la redacción de un nuevo Plan General, ya que puede estructurarse como una revisión parcial del mismo (modificación estructural), para más tarde afrontar el conjunto de desarrollos puntuales de menor rango a través del resto de figuras de Ordenación Urbana de menor rango. Hasta cierto punto es un mecanismo más para hacer realidad ese objetivo denominado como "crecer hacia adentro" o mejor dicho "crecer en el interior". Un procedimiento de actuación más sostenible que el de ampliar los límites de la ciudad con crecimientos adicionales ${ }^{1}$. Últimamente muy reiterado por quienes intervienen en el mundo del urbanismo, y que se justifica en un contexto cada vez más sensible hacia temas de primer orden como es la intensidad de uso del suelo urbanizado de aquellas ciudades que demandan un crecimiento justificado de su parque residencial.

\footnotetext{
${ }^{1}$ De hecho, los últimos intentos en la ciudad de Vitoria-Gasteiz están yendo en la línea contraria, intentando la desclasificación de suelo (trasladando y concentrando derechos edificatorios en áreas ya urbanizadas) para generar reservas a muy largo plazo, o incluso "devolver" suelo al entorno natural. Por ejemplo, en el Sector 14 "Olaran" de Salburua, Ensanche XXI 2020.
}

ACE, X (XX) CC BY-ND 3.0 ES | UPC Barcelona, España | Otros procedimientos de intervención en la ciudad contemporánea: sobre la re-densificación. DOI: http://dx.doi.org/10.5821/ace.15.45.9527 
Pero previamente sería necesario erradicar la idea simplista de identificar re-densificación con el hecho de llenar los huecos disponibles en la trama urbana, permitir un mayor número de unidades residenciales, o el de subir más alturas sobre las inicialmente permitidas por el planeamiento con el fin de incrementar simplemente la compacidad física. Aspectos extremadamente difíciles de asimilar de cara a su aceptación inicial. En primer lugar, por su simpleza puramente cuantitativa, y en segundo porque todo cambio en la morfología de la ciudad siempre es mirado con recelo por sus habitantes más afectados. Y especificar que se trata de un incremento de edificabilidad física sin una alteración del perímetro, que conlleva el consiguiente incremento de las dotaciones y equipamientos especificados en la ley de aplicación vigente, y que como es lógico, modifican la ocupación del suelo dentro del ámbito. También sería importante reclamar su grado de complejidad escalable, que puede llegar hasta el nivel de modificar tejidos y trasladar usos, mezclando, optimizando, corrigiendo y racionalizando su explotación. Persiguiendo una mejor interacción entre los distintos distritos que ha de evitar desequilibrios. Pero siempre con el compromiso de respetar la verdadera identidad de los entornos en donde opera, y defender por último su posibilidad legal en el marco vigente, tal y como lo demuestra el caso elegido, que no sin dificultades en el largo camino de su materialización real (con una nueva ley de aplicación, Ley 2/2006, durante su proceso de desarrollo), se enfrenta en la actualidad a un proceso de recapitulación que está desembocando en una reedición de lo ya previamente acontecido.

Todos podemos adivinar las ventajas derivadas de las apreciaciones anteriores; repetidas de forma común como obviedades encaminadas a apuntalar el concepto de un urbanismo más equilibrado desde la aproximación que postula un menor consumo de recursos, así como un aprovechamiento más intenso de los existentes. E implementar, dentro de la praxis de la disciplina, un grado más de compromiso cuando se abordan suelos ya clasificados. Además, sería interesante recordar que este tipo de intervenciones también son capaces de incrementar esas condiciones de diversidad que enriquecen a una ciudad, y que parecen cada vez más olvidadas en virtud de otros postulados tecnológicos.

En cualquier caso y desde un punto de vista crítico sobre el ejemplo elegido, es necesario reflejar desde un comienzo una enumeración de sus inconvenientes, que son precisamente el motivo de muchos de los conflictos experimentados durante su aplicación.

1. La oposición vecinal latente; fundamentalmente por falta de información, participación desde el origen y entendimiento de los objetivos, factores por los que tiende a sospechar de las decisiones de los representantes políticos.

2. La necesidad de una visión global e integrada de la forma urbana por parte de la Administración Local. Todavía más preocupada por actuaciones cortoplacistas y de carácter puntual, pero de gran repercusión mediática (Moix, 2010). Generalmente ajenas a la consideración de la ciudad en todo su conjunto.

3. El conflicto siempre latente entre el interés público y los intereses particulares, motivados éstos últimos por los propietarios del suelo, y que sorprendentemente coinciden en este caso con los de la propia Administración². Al olvidar que la ciudad debe configurarse por y para los ciudadanos (Gehl, 2010), evitando correcciones improvisadas a posteriori.

\footnotetext{
2 Por ejemplo y tras los momentos de crisis en los que se escribe este texto, es curiosa la coincidencia entre propietarios particulares y representantes tanto técnicos como políticos de la administración acerca de la supresión de reserva de suelo destinado a viviendas unifamiliares: Hace bien poco fuertemente demandado. No se trata por lo general de que estén preocupados por un uso indiscriminado del suelo, o por el resultado morfológico de la ciudad. Más bien los primeros intentan reconvertir un producto de complejo desarrollo y que no venden con facilidad, mientras que los segundos acceden a lo que sea con tal de mantener cierta actividad económica en el campo de la promoción inmobiliaria y los entornos económicas que mueve.
}

ACE, X (XX) CC BY-ND 3.0 ES | UPC Barcelona, España | Otros procedimientos de intervención en la ciudad contemporánea: sobre la re-densificación. DOI: http://dx.doi.org/10.5821/ace.15.45.9527 
4. La opción equivocada de re-densificar tan solo en nuevos ámbitos urbanos todavía sin consolidar, (si bien es perfectamente posible hacerlo en los existentes) creando elementos urbanos dispares y manifiestas discontinuidades de la intensidad edificada. De hecho, el ámbito ideal para una re-densificación exenta de conflictos lo constituirían aquellos suelos urbanos hace ya tiempo consolidados, que así podrían ser renovados. O en su caso, y como alternativa, los totalmente nuevos y en absoluto consolidados, que de este modo podrían encontrar su densidad adecuada. Pero a ser posible nunca en los semiconsolidados (urbanizados, pero tan solo parcialmente edificados), fundamentalmente por dos razones: Por un lado, las quejas de los nuevos vecinos por el repentino y prematuro cambio de sus expectativas respecto del entorno inmediato. Y por el otro, al ridículo que puede pesar sobre la administración competente debido a su falta de previsión a corto plazo.

Una vez introducido el concepto y su problemática general, cabe enfocar más a fondo el tema según el siguiente índice que se acompaña:

- Posibles mejoras en el urbanismo actual mediante el uso de la herramienta de la re-densificación

- Análisis de la situación actual. Comparación crítica entre algunos datos generales de interés y aspectos puntuales a considerar para este caso particular

- Principales objetivos urbanísticos de una re-densificación

- El caso particular de los suelos semiconsolidados. La gran dificultad de la re-densificación

- Fases del proceso

- Gráficos comparativos del resultado final

- Conclusiones

El cual sirve como marco metodológico de referencia de los diferentes comentarios razonados sobre el hilo argumental, para finalizar con los datos comparativos y las imágenes del caso de estudio elegido.

\section{Posibles mejoras en el urbanismo actual mediante el uso de la herramienta de la re-densificación}

Ya hemos defendido que la re-densificación como incremento de la edificabilidad física, es una de las consecuencias más razonables de esa nueva idea del urbanismo sostenible que supone el ya citado "crecer en el interior". Sin embargo, y hasta el caso de Vitoria-Gasteiz, ha sido poco utilizado en el contexto del Estado Español ${ }^{3}$ debido fundamentalmente a su carácter novedoso, en contraste con ejemplos conocidos del continente americano ( $p$-ej. El de Guadalajara, México ${ }^{4}$ o Bogotá, Colombia ${ }^{5}$.

\footnotetext{
${ }^{3}$ Al margen de procesos puntuales y casi orgánicos desarrollados a lo largo del tiempo como por ejemplo la colmatación de los espacios interiores de los patios de manzanas en el ensanche Barcelonés de I. Cerda de 1859. O los intentos incipientes de las federaciones de vecinos de Zaragoza a partir del 2009 que han sido replicados en otras ciudades españolas con el apoyo municipal correspondiente.

${ }^{4}$ Que ha afectado a más de $2.000 \mathrm{Ha}$. Consultar el Foro "Densificación, Infraestructura y Vivienda Social en la zona metropolitana de Guadalajara” y https://guadalajara.gob.mx/comunicados/nueva-politica-redensificacion-guadalajara. En México ha calado esta herramienta tal y como lo demuestra el programa de re-densificación urbana que ha sido presentado por el CIDS (Centro Integral de Desarrollo Social) en una exposición en la sede central del INFONAVIT y en el libro homónimo que reúne treinta y dos proyectos seleccionados a partir de una convocatoria pública (David Penchyna y Carlos Zedillo, 2018)

${ }^{5}$ Descrito con detalle en la literatura académica de referencia (Rincón Avellaneda, 2004)
}

ACE, X (XX) CC BY-ND 3.0 ES | UPC Barcelona, España | Otros procedimientos de intervención en la ciudad contemporánea: sobre la re-densificación. DOI: $\underline{\text { http://dx.doi.org/10.5821/ace.15.45.9527 }}$ 
Tras un rápido análisis previo del estado del arte, éstos son algunos aspectos obvios derivados de su aplicación, y que han sido concretados en diferentes foros especializados ${ }^{6}$.

- Reduce el consumo indiscriminado de un bien no renovable como es el suelo.

- Incrementa la densidad y la edificabilidad física necesarias para mejorar la calidad urbana con criterios de sostenibilidad, sin reducir la ratio de equipamientos fijados por Ley.

- Permite ajustar el tamaño y tipología de las viviendas a la cada vez más variada demanda social existente.

- Puede generar desequilibrios con otros distritos de la ciudad e impactos no deseados, por lo que conviene evaluar de forma previa y cuidadosa sus consecuencias.

- Puede adaptar el planeamiento a los requerimientos establecidos por las leyes autonómicas que buscan cada vez más una adecuada densidad necesaria para hacer ciudad ${ }^{7}$. En este caso particular a la Ley 2/2006 de Suelo y Urbanismo del País Vasco.

Estos criterios de partida, en función del estado de desarrollo de cada Área o Sector ${ }^{8}$, así como de su localización, exigen lógicamente una sistemática de aplicación diferenciada.

Tras una previa revisión obligada de la figura de planeamiento general, puesto que se trata de modificaciones estructurales, es necesario el desarrollo de figuras urbanísticas específicas con el fin de operar con eficacia y libertad suficiente a la hora de incrementar densidades e intensidades urbanas $^{9}$. Así, parece lógico aplicar las Actuaciones de Dotación para el suelo urbano consolidado, mientras que para los suelos urbanizables resulta más eficaz la modificación integral de los Planes Parciales correspondientes.

\section{Análisis de la situación actual. Comparación crítica entre algunos datos generales de interés y aspectos puntuales a considerar para el caso particular de Vitoria-Gasteiz}

La existencia de una serie de circunstancias propias de la práctica urbanística que atienden a aquellos cómputos globales que superan ampliamente los mínimos legales para el conjunto del suelo urbano debieran someterse a un juicio crítico permanente. Sus consecuencias inmediatas aminoran cíclicamente el valor de las reservas de suelo urbanizable, propiciando su colmatación en un plazo relativamente breve, o cuando menos no previsible con precisión. Hecho que requiere de una

\footnotetext{
${ }^{6}$ Como por ejemplo en el I Encuentro Internacional de Urbanismo Sostenible celebrado en Vitoria- Gasteiz marzo / abril de 2011. O en las II Jornadas Europeas sobre Eficiencia Energética y Sostenibilidad en arquitectura y urbanismo, dentro de los XXX Cursos de Verano - XXIII Cursos Europeos organizados por la UPV-EHU en San Sebastián, también en 2011.

${ }^{7}$ Y en este sentido parece que el número de $75 \mathrm{viv} / \mathrm{Ha}$, heredado a lo largo de las diferentes Leyes del Suelo estatales, ha llegado a su obsolescencia. Toda vez que como densidad neta no es capaz de generar la intensidad urbana necesaria para configurar la ciudad compacta que reconocemos en la Europa Meridional, la cual supera con creces este número. 8 El término Sector se utiliza en el artículo porque las figuras urbanísticas necesarias para la consecución de la redensificación pasan eventualmente por la modificación de los diferentes Planes Parciales de cada uno de los "sectores", a pesar de que en realidad debieran denominarse "áreas" cuando se trata de suelo urbano. La práctica urbanística habitual sin embargo utiliza por extensión el término original de "sector".

${ }^{9}$ Así en los Sectores muy avanzados en su ejecución, con la urbanización terminada o en fase de remate, y la edificación en su mayor parte construida y habitada, parece lógico que se intervenga a través de Actuaciones de Dotación, según regula en su Art. 137 de la Ley 2/2006 de Suelo y Urbanismo del País Vasco para hacerlo en suelo urbano.
}

ACE, X (XX) CC BY-ND 3.0 ES | UPC Barcelona, España | Otros procedimientos de intervención en la ciudad contemporánea: sobre la re-densificación. DOI: http://dx.doi.org/10.5821/ace.15.45.9527 
constante revisión sistemática de nuestros Planes Generales, independientemente de su vigencia prevista $^{10}$. Entre estas circunstancias podemos señalar:

- La baja densidad neta. Todavía inferior a las 75 viv/Ha que, tras la Ley del Suelo del 56, y su posterior revisión del 75, fue referencia del planeamiento nacional de los años 70 y 80, y se adoptó en los sectores desarrollados previamente a la aprobación de la Ley del Suelo del País Vasco vigente 2/2006, que intenta incrementar esta ratio de forma significativa. Se trata de un valor que todavía subyace como referencia en la práctica totalidad del planeamiento estatal, independientemente de la intensidad y la naturaleza del tejido urbano que se trate, sin estar muy claro su origen ni su justificación (Fariña, 2014).

- La gran cantidad de zonas verdes de dimensión generosa, consecuencia de incluir dentro de los nuevos sectores tanto parcelas calificadas pormenorizadamente, como otras zonas verdes de sistema general. Cuando además habría que añadir a éstas las zonas verdes de menor entidad, las áreas de juego locales, los espacios libres, etc.

Es interesante darnos cuenta de que la dotación de zonas verdes en los nuevos ensanches de nuestras ciudades planificadas de tamaño pequeño o medio resulta por lo general muy generosa, y cometeríamos un error al seguir incrementándola. Máxime cuando el entorno inmediato de estos núcleos urbanos supone muchas veces un espacio natural privilegiado muy apreciado por la población, de indudable valor ambiental, y nulo costo de conservación.

La Ley 2/2006 del País Vasco, así como la Ley Estatal, establecen la dotación mínima de Parques Urbanos, independiente de las Zonas Verdes Locales, en $5 \mathrm{~m}^{2} /$ habitante. Tomando como ejemplo la ciudad de Vitoria-Gasteiz, cuyo número de habitantes total arrojaba al comienzo del proceso de redensificación un total de 239.361 habitantes en todo el término municipal (censo del 2010), cumpliría el estándar establecido para una población de 1.120.039 habitantes. Resultando una dotación por habitante de 15,21 $\mathrm{m}^{211}$, sin computar las zonas verdes de Sistema Local (es decir, ya más que el triple del mínimo legal). Por lo que una reducción moderada no debiera ser motivo de conflicto a la vista de estos resultados.

- El sobredimensionamiento de las parcelas calificadas como Equipamiento de Sistema General, que la mayor parte de las veces se solapan funcionalmente (pues sirven para lo mismo), y duplican las reservas determinadas como Sistemas Locales.

- Si se observa con detenimiento los datos (bien sean numéricos o gráficos coloreados), en general los equipamientos públicos ocupan, agrupando los de Sistema General y Local, la misma superficie que las parcelas residenciales colectivas, lo que resulta cuando menos competitivo en términos de figura-fondo ${ }^{12}$, pues no deja de ser un espacio "al servicio" de las viviendas; que es el uso preferente de los sectores residenciales analizados.

- La consecuente reducción correctiva de los viarios (tanto de Sistema General como Local), motivada sin duda por la necesidad de alojar reservas de zonas verdes y de equipamiento. Pero

\footnotetext{
${ }^{10}$ Sirva como ejemplo la situación en Euskadi, en la que utilizando como fuente el Inventario que elabora el Departamento de Medio Ambiente y Política Territorial del Gobierno Vasco podemos observar que de acuerdo con la actualización de fecha 01-04-2013 existen superposiciones de planeamiento vigente y en revisión hasta en 13 municipios del TH de Álava, en 25 del TH de Gipuzkoa, y en 35 del TH de Bizkaia. Casi el 30\% de los 251 municipios existentes 10. http://www.ingurumena.ejgv.euskadi.net/r49-578/es/

11 Ocupando un lugar intermedio entre las capitales europeas (Berlín, Paris, Londres, o Madrid) dato de mayor transcendencia máxime cuando se trata de una ciudad de tamaño medio rodeada de un entorno natural de alta calidad, con fáciles accesos al territorio y sin las necesidades de otras grandes urbes.

12 Adaptando el criterio utilizado por C. Rowe y F. Koetter en su "Ciudad Collage" Edición en español GG reprints $1981-98$. Edic. original 1978 para este particular.
}

ACE, X (XX) CC BY-ND 3.0 ES | UPC Barcelona, España | Otros procedimientos de intervención en la ciudad 
que acaban por configurar grandes manzanas en donde se reduce la posibilidad de una conectividad alternativa intermedia, ya sea peatonal o rodada.

La investigación realizada aumentando los parámetros lucrativos demuestra que existe un punto de inflexión a partir del cual por mucho que se incremente la densidad residencial, no se incrementa la compacidad del modelo. Es decir, el sistema colapsa, y la necesaria reserva de dotaciones y zonas verdes libres que demanda la ley, exige concentrar las viviendas en parcelas residenciales cada vez más pequeñas, generando edificios en altura y desperdigados que no logran formar ciudad. Asimismo, la superficie media de las viviendas con las que se trabaja parece desmesurada y demasiado uniforme, sin ofrecer una diversidad de programa y tipología acorde con las demandas de la sociedad actual.

Desde las figuras de planeamiento vigentes existe una manifiesta inflexibilidad a la hora de establecer un abanico más amplio respecto de su superficie, dificultando el ajuste la repercusión del suelo y los siempre temidos gastos de urbanización por vivienda. Esto provoca que la inmensa mayoría de las viviendas respondan al tradicional programa de 3 dormitorios dobles; 2 baños; cocina y EstarComedor. Similar desde hace por lo menos medio siglo. Y ajeno a la composición familiar que ha experimentado una transformación muy importante en los últimos años ${ }^{13}$. Es por ello, que independientemente de un incremento de la edificabilidad física, debe permitirse la reducción del tamaño de la vivienda y un consiguiente aumento del número de unidades, de manera que se adecue mejor a la diversidad de la demanda social. Un decremento del tamaño medio de la vivienda adaptándolo a las necesidades reales de la población demandante tiene dos consecuencias positivas añadidas. Por un lado, la reducción de su precio; factor decisivo teniendo en cuenta la difícil situación económica que durante un cierto tiempo padeceremos, acompañada de una inseguridad del empleo y un difícil acceso al crédito. Por el otro, la optimización del espacio habitable. Con el mismo volumen e idénticos costes de urbanización y de mantenimiento, se pueden satisfacer las necesidades de alojamiento de un mayor número de personas. Limitando quizá la tipología unifamiliar tradicional, para sustituirla por otras alternativas residenciales colectivas de carácter diverso que actualmente están floreciendo (cooperativas, co-housing, etc.).

De esta manera, y en el caso concreto de los nuevos desarrollos de la ciudad de Vitoria-Gasteiz, las parcelas residenciales presentan una uniformidad tipológica y de edificabilidad prácticamente similar, y tal y como ya se ha avanzado, su ocupación no suele alcanzar un porcentaje razonable del ámbito total. En la práctica, no llegan al 50\% tratándose de Sectores con Calificación y Uso predominante residencial, algo muy distinto de lo que ocurre en países con una zonificación más clara como puedan ser los del Norte de Europa, en los que la naturaleza de cada tejido urbano es claramente identificable. Esta circunstancia tiene una consecuencia formal desastrosa para la ciudad: Aun alcanzando una densidad muy baja, la altura media edificable de los bloques resulta siempre muy alta, lo que configura un urbanismo de grandes bloques y vacíos. El Urbanismo históricamente denominado como "the city of towers in the park" pero irónicamente calificado como "the city of towers in the parking" (Rowe y Koetter, 1978) por sus nefastos resultados ${ }^{14}$.

El modelo urbano ya conocido al que estos parámetros nos avocan no es otro sino el propio de una ciudad con las siguientes características perniciosas:

\footnotetext{
${ }^{13}$ Según datos del Departamento de Vivienda del Gobierno Vasco, en 2.009, la media de personas por familia era de 2,68 en el País Vasco, (Fuentes: Censos de Población y Viviendas, 1970, 1991 y 2001. Eustat, INE. EPDS 2008 y Avance de CMT 2009. Departamento de Empleo y Asuntos Sociales) con tendencia a la baja y ya cerca de las tasas propias de los países del centro de Europa.

${ }^{14}$ Esta conocida cita expresada en "Collage City" aparece también en "The New City: Architecture and Urban Renewal," pp. 87-96
}

ACE, X (XX) CC BY-ND 3.0 ES | UPC Barcelona, España | Otros procedimientos de intervención en la ciudad contemporánea: sobre la re-densificación. DOI: http://dx.doi.org/10.5821/ace.15.45.9527 
- Escasa compacidad de la trama urbana, y exceso de espacios libres públicos sin función determinada; muy costosos en su urbanización y conservación, y que caracterizan el denominado como "urban sprawl" no solo en las grandes ciudades (Arellano y Roca. 2012).

- Sensación inevitable de inseguridad, porque los espacios públicos sobredimensionados rebasan la escala humana; y resultan fríos, impersonales, e incluso peligrosos.

- Discontinuidad de la trama urbana, la cual genera vacíos que disuaden y dificultan los desplazamientos peatonales de proximidad. Sin ofrecer como alternativa una diversidad ni el tipo de vivienda, ni en el carácter del espacio público.

- Dificultad de funcionamiento racional e intensivo del transporte público por falta de densidad humana, y exceso de trayectos largos sin apenas paradas.

- Estimulación de la necesidad del uso del vehículo privado; con las repercusiones negativas de contaminación, congestión, necesidad de aparcamientos, etc., que todo ello genera ${ }^{15}$.

En resumen, una ciudad impersonal, cara de construcción y de mantenimiento. Buena quizá para las estadísticas, pero hostil para el ciudadano, y en la que, a pesar de la existencia de gran cantidad de espacio público, las relaciones humanas quedan muy limitadas. Nada que ver con el concepto tradicional de la ciudad mediterránea cuya compacidad debe ser entendida como "atributo de urbanidad" (Górgolas, 2018). En la que el dominio público, aunque reducido e incluso a veces tortuoso, es el protagonista como escenario de unas ricas relaciones sociales.

Pero además de lo expuesto, los crecimientos esponjados y de baja densidad requieren una mayor ocupación del territorio, con la invasión indiscriminada de suelo natural. Bien escaso y no renovable, que tenemos la obligación y responsabilidad de administrar con prudencia. Sin embargo, este modelo de desarrollo que resulta ajeno a la identidad de nuestras ciudades todavía es considerado por muchos sectores de población como el paradigma del bienestar en contra de la ciudad compacta (Bohigas, 2004). Con sus grandes espacios abiertos, ajardinados y luminosos. Aquéllos a los que, independientemente de los problemas anteriormente citados que generan, no están dispuestos a renunciar. Cueste lo que cueste.

Podemos afirmar que esta situación contradictoria responde en cierta manera a un modelo de civilización del derroche, de la insolidaridad, del desarrollo injustificado (sostenido, pero no sostenible). Y todo ello para obtener una calidad de vida que se ha manifestado claramente insatisfactoria; representativa del conflicto latente que existe dentro de la sociedad actual entre lo deseado y lo razonable.

\section{Principales objetivos urbanísticos de una re-densificación como la realizada en Vitoria-Gasteiz}

Tras las consideraciones anteriores, quedan claros los objetivos urbanísticos que debiera conseguir una revisión de planeamiento que trata de encontrar una densidad adecuada. Aquellos con los que en principio debieran estar de acuerdo todos los agentes implicados:

\footnotetext{
15 La aparición del coche eléctrico, tratada como el nuevo maná urbano de la movilidad privada, puede que solucione parcialmente los problemas de contaminación. Pero el estorbo que supone una máquina como el coche, por su amplitud y condiciones de manejo, seguirá reclamando un diseño específico de nuestras vías urbanas para el vehículo rodado, casi siempre ajeno a los requerimientos del peatón.
}

ACE, X (XX) CC BY-ND 3.0 ES | UPC Barcelona, España | Otros procedimientos de intervención en la ciudad contemporánea: sobre la re-densificación. DOI: http://dx.doi.org/10.5821/ace.15.45.9527 
- Obtener una ciudad más densa, diversa y compacta. Acorde con lo que hoy se denomina un desarrollo sostenible; aquél que limita fundamentalmente el consumo de suelo, sin rupturas traumáticas, discontinuidades, ni vacíos dentro de su trama. Con unos espacios públicos que fomentan los desplazamientos peatonales y ciclables, así como un transporte público eficaz. Y un ajuste equilibrado entre el incremento de la densidad residencial y la reducción ajustada de los espacios libres y las zonas verdes, las cuales deben estar correctamente entrelazados. Es decir, una ciudad mejor diseñada bajo "acciones formalizadoras y estructurantes" (Terán, 2009 p. 65)

- Mejorar la calidad del espacio público de forma que recupere las virtudes tradicionales de la ciudad compacta, revitalizándolo con una mayor presencia peatonal dedicada a actividades varias, sobre recorridos alternativos, y en horarios diferentes ${ }^{16}$.

- Reajustar el dimensionamiento de los equipamientos públicos evitando solapamientos y reservas innecesarias, la dotación justa de aparcamientos en superficie, y la creación de parcelas para nuevos usos flexibles como puedan ser los Alojamientos Dotacionales ${ }^{17}$.

- Reducir el tamaño medio de las viviendas como forma de adecuarlas mejor a la demanda social real; fundamentalmente a sus posibilidades económicas. Permitiendo así satisfacer las necesidades de más población con los mismos recursos.

- Reconsideración de las tipologías residenciales, reduciendo las de tipo unifamiliar o las comunidades valladas. Aumentando la representatividad de las viviendas protegidas, bien sean sociales o tasadas, como protagonistas indiscutibles del escenario urbano definitivo, de acuerdo en este caso con las exigencias de la Ley 2/2006. Pero sin olvidar el daño que también ejercen otras tipologías muy comunes como los condominios; esos grandes enemigos de lo urbano que sin embargo siguen siendo considerados como un referente de calidad de vida.

Estos objetivos son replicables para la mayor parte de las ciudades intermedias del Estado Español, por lo que la experiencia de este caso particular puede resultar útil para otros contextos similares.

\section{El caso particular de los suelos semiconsolidados: La gran dificultad de la re-densificación}

Tras la experiencia práctica experimentada, parece importante considerar otros aspectos significativos cuando se trata de una intervención sobre una parte de la ciudad en fase de construcción, e inserta en una trama ya consolidada con la que interactúa. Posiblemente el caso más problemático de cara al desarrollo fluido de la misma, cuyo proceso de crecimiento y construcción vital no se desea paralizar ni penalizar en virtud de una serie de posturas enfrentadas.

Dicho de otro modo, la ordenación pormenorizada en suelos semiconsolidados ${ }^{18}$, también es susceptible de modificarse (aunque no sin problemas) a pesar del consiguiente recelo de sus habitantes. Adaptándola a los nuevos criterios de re-densificación propuestos sin detrimento de su

\footnotetext{
${ }^{16}$ Recordando alguna de las condiciones de la diversidad urbana defendidas por Jane Jacobs en su "The Death and Life of Great American Cities” 1961. Edición en español, Capitán Swing 2011.

17 Una de las grandes novedades como consecuencia de la adaptación del planeamiento a la última Ley 2/2006, de Suelo y Urbanismo del País Vasco. En principio un uso residencial dirigido hacia los sectores sociales más vulnerables como son los jóvenes y los mayores, pero que pueden tener otros enfoques en un futuro.

18 SUNC según la Ley 2/2006, que concretamente en este caso se identifican con aquéllos prácticamente urbanizados y con la urbanización ya recibida, pero no colmatados por la edificación. Debido al proceso paralelo de desarrollo de suelos urbanizables afectados también por la re-densificación durante este caso concreto, y en proceso de urbanización, pero sin la clasificación definitiva de SU, se utiliza este término que de alguna manera abarca la afecciones sobre ambos.
}

ACE, X (XX) CC BY-ND 3.0 ES | UPC Barcelona, España | Otros procedimientos de intervención en la ciudad contemporánea: sobre la re-densificación. DOI: http://dx.doi.org/10.5821/ace.15.45.9527 
carácter ni de su unidad. Con el objeto de tratar de mejorar su integración sin rupturas, ni barreras, ni vacíos con el entorno urbano colindante. Pero no sin observar algunas precauciones.

Para ello, parece conveniente mantener la ordenación urbanística general vigente en los ámbitos afectados por cualquier tipo de revisión. También y no menos importante su identidad, como reflejo y punto de apoyo de sus habitantes. Permitiendo asimismo aprovechar en todo lo posible la urbanización existente. En la que únicamente deberán introducirse mínimas modificaciones por la necesidad de dotación de servicios urbanísticos a parcelas de nueva creación, desvíos de algún ramal o colector secundario, u otras intervenciones menores. En algún caso también puede ser necesaria la apertura de algún viario local, así como la ampliación o reducción de plazas de aparcamiento en superficie. Pero en general, estos costes se verán compensados por la reducción de superficies infrautilizadas. Así como por el incremento de las parcelas residenciales que a su vez tenderán a minorar las superficies susceptibles de urbanizarse. Siendo todos ellos hechos fácilmente comprendidos y asimilados por sus habitantes.

Tan solo en casos excepcionales, y tras analizar en profundidad su conveniencia y necesidad, será necesaria la intervención sobre parcelas residenciales ya edificadas, o que cuenten con un proyecto arquitectónico en tramitación. Por lo tanto, una Revisión Parcial del Planeamiento General motivada por una re-densificación en este tipo de suelos debiera, de manera general, procurar:

- Respetar los criterios de ordenación urbanística general de los Planes Parciales o MOAS (Mantenimiento de la Ordenación Actual) del ámbito (red viaria, áreas residenciales, etc.)

- Mantener la urbanización existente y en ejecución en la medida de lo posible (colectores principales, criterios de servicios urbanísticos, etc.)

- Adaptarse al ritmo de ejecución o de intensidad de uso del ámbito de actuación. Sin necesidad de paralizaciones que impidan cumplir los compromisos adquiridos, o restar vida a los ámbitos en los que se actúa.

- Conservar el carácter propio de cada Sector o ámbito de actuación, optimizando el uso del suelo sin alterar su relación con los colindantes, ni su papel dentro del conjunto de la ciudad.

Previamente habría que tener en cuenta los condicionantes derivados de:

- Edificaciones ejecutadas o en fase de construcción, de acuerdo con el planeamiento parcial vigente.

- Parcelas comprometidas, bien destinadas a equipamientos y cedidas a otros órganos de la administración pública, o bien adjudicadas a través de concursos o proyectos estratégicos.

- Algunos otros condicionantes físicos que pudieran existir referentes a la urbanización; redes de servicios urbanísticos generales de nivel ciudad, servidumbres, afección a acuíferos, lugares protegidos, etc.

- Impactos sobre el resto de la ciudad, especialmente aquéllos negativos que puedan generarse por el desplazamiento de sectores determinados de población y actividades, vaciando barrios existentes o modificando significativamente su naturaleza.

- Y lógicamente, todos aquellos propios del enlace con los ámbitos perimetrales externos; los de tipo físico y paisajístico (evaluación de su impacto ambiental), así como el eterno problema del remate de los tejidos urbanos en su encuentro con el entorno no urbanizado (bordes y límites de ciudad). 
Esto permite extraer los datos, parámetros y propuestas que deben recogerse y formar parte de un trabajo que no acaba en el planeamiento. Convirtiéndose en fuentes para futuras actuaciones, pues constituyen determinaciones de carácter estructural con el fin de legitimar intervenciones susceptibles de discusión y participación popular bajo un enfoque integrado y de buenas prácticas (De Gregorio Hurtado, 2019). Es importante estudiar en cada ámbito las posibilidades que su ordenación urbanística ofrece bajo los programas vigentes, encaminadas a lograr los objetivos iniciales y comúnmente planteados de:

- Alcanzar una densificación adecuada que garantice complejidad urbana, incrementando la intensidad edificatoria y de ocupación de las parcelas residenciales con diferentes usos, así como la revisión de las tipologías adaptadas a las demandas reales de la sociedad.

- Mejora de la calidad del espacio público, evitando rupturas o espacios vacíos en la trama de los principales itinerarios urbanos. Incrementando su continuidad y complejidad de cara a procurar que haya todo tipo de gente en la calle para facilitar posibles encuentros casuales.

- Reajustar el dimensionamiento de los Equipamientos y de las Zonas Verdes, de manera que siempre se garantice la mínima dotación adecuada sin caer en su exceso; optimizando el rendimiento del suelo ocupado.

- Adaptarse a la legislación aplicable; en este caso particular a las exigencias de la Ley del Suelo 2/2.006.

Por lo tanto, es necesario estudiar detenidamente los planeamientos vigentes, sus relaciones formales y funcionales, las razones de su ordenación urbanística, así como su grado de ejecución y vinculación. Sin olvidar que la coparticipación en incluso la "co-elaboración" de la ciudadanía es un factor capital que se ha de tener en cuenta desde un principio. Pudiendo así deducir las posibilidades de modificación que ofrecen en relación con los objetivos pretendidos, pero sin generar otro tipo de conflictos.

Planteadas las modificaciones, tanto en calificación pormenorizada como en cuantificación de aprovechamientos edificables, y habiendo revisado el cumplimiento de las dotaciones de Equipamiento, Espacios Libres-Zonas Verdes y Alojamientos Dotacionales, finalmente habrá que comprobar y evaluar la fase final de la intervención constructiva. Tanto en cuanto a las obras de urbanización necesarias para garantizar acometidas y servicios soterrados a las nuevas parcelas creadas o a las existentes incrementadas, como respecto a la dotación de aparcamientos y servicios en superficie adscritos a la red viaria.

La gran ventaja es que, dado el carácter urbano, aunque no colmatado de los suelos tratados, y la no existencia de Proyectos de Concertación o Reparcelación ${ }^{19}$, no es necesario en estos casos acordar fórmulas de compensación. Evitando el establecimiento de coeficientes de homogeneización o figuras de reparto similares que normalmente exigen un prolongado periodo de negociación antes de un acuerdo.

\footnotetext{
19 Según los cuales a los propietarios particulares de terrenos o cesiones dentro del ámbito se les adjudica aprovechamiento lucrativo localizado en parcelas de nueva creación en función de unos coeficientes de homogeneización previamente establecidos y aprobados por todos de cara a un reparto equitativo.

En este caso garantizado por el Convenio para las ampliaciones de la Ciudad al Este y al Oeste en Salburua y Zabalgana, aprobado en Pleno Municipal el 1 de agosto del 2008, y que tiene como objetivo no solo dicho reparto equitativo entre ayuntamiento y particulares, sino la autofinanciación de un proceso que todavía se encuentra activo.
}

ACE, X (XX) CC BY-ND 3.0 ES | UPC Barcelona, España | Otros procedimientos de intervención en la ciudad contemporánea: sobre la re-densificación. DOI: http://dx.doi.org/10.5821/ace.15.45.9527 


\section{Fases del proceso}

Tras la experiencia adquirida, y a modo de manual operativo de la experiencia extraída del caso de estudio que nos sirve de guía, cabe plantear el siguiente procedimiento metodológico. El contenido de cada fase aplicable puede ser el siguiente:

\subsection{Fase I. Estudio de Alternativas}

Consistente en elaborar las propuestas de la modificación de ordenación en los Planes Parciales o MOAS de los diferentes sectores, en relación con los objetivos inicialmente planteados. Este apartado debe comprender:

- Un análisis pormenorizado de la ordenación vigente en cada Sector o Área de planeamiento, y de sus parámetros fundamentales. Así como su contexto y relación con el resto de la ciudad.

- El estado de desarrollo de cada ámbito, tanto en cuanto a obras de urbanización, como en cuanto a edificación (materializada o en proceso de). Así como posibles compromisos de cesión de parcelas de equipamiento público a otros órganos de la administración.

- Las relaciones urbanísticas con el resto de los sectores, consolidados o no, así como su ámbito de afección perimetral. Siendo sensibles a interacciones presentes y futuras de todo tipo (no solo físicas).

- La elaboración de alternativas de modificación. Con el objeto de posibilitar un proceso de participación ciudadana más abierto y plural sobre unas bases concretas.

- Un breve desglose explicativo de las alternativas elaboradas. Atendiendo a la cuantificación de calificaciones urbanísticas, los aprovechamientos definitivos, las principales intervenciones sobre la ordenación urbanística vigente tanto sobre el espacio público y su imagen, como la afección respecto de la urbanización. Además de la evaluación económica de los costes derivados de su implantación.

- Un calendario realista del procedimiento jurídico y administrativo preciso para su tramitación y posterior ejecución.

- La elaboración del prescriptivo documento de "Estudio de Alternativas" definitivo para su trámite legal de información pública.

\subsection{Fase II. Revisión Parcial del Plan General a través de los trámites de exposición pública y aprobaciones específicos}

El documento de "Revisión Parcial del Plan General", debe reflejar las alternativas que sean elegidas como más adecuadas de entre las presentadas en la Fase I para cada ámbito. Con todas las matizaciones o indicaciones que en su caso procedan. Así se elabora el documento de "Avance de Planeamiento", destinado a su exposición y debate durante el periodo de participación pública. Dada su principal finalidad, debe tratarse de un documento que defina los criterios, objetivos, y en su caso alternativas de la propuesta de revisión. Con el fin de que cumpla su cometido, debe ser claro e inteligible por un público sin conocimientos profundos de urbanismo y legalidad urbanística. Completo, pero a la vez sencillo, con el claro objeto de que toda persona interesada sea capaz de entenderlo. El "Avance de Planeamiento" también estará sujeto a las determinaciones de la Ley 21/2013, de Evaluación Ambiental, incluyendo cuantos documentos ambientales sean perceptivos.

ACE, X (XX) CC BY-ND 3.0 ES | UPC Barcelona, España | Otros procedimientos de intervención en la ciudad contemporánea: sobre la re-densificación. DOI: http://dx.doi.org/10.5821/ace.15.45.9527 
Paralelamente debe estudiarse la mejor forma de realizar una información pública amplia, transversal y continuada. Fomentando en el mayor grado posible la coparticipación ciudadana que debiera de haber surgido ya en las primeras fases del proceso. Elaborándose, si es preciso, todo tipo de documentación legible (paneles, folletos explicativos, exposiciones, etc.) que faciliten dicha participación en cada una de las fases, y aseguren la comprensión de la propuesta urbanística por parte de los diferentes consejos ciudadanos. Y es que el proceso de participación pública ha ido cobrando mayor importancia a medida que la sociedad ha asumido los principios del funcionamiento democrático. Hoy en día todo ciudadano tiene derecho a conocer y expresar su opinión sobre las propuestas y transformaciones urbanísticas que afectan a su ciudad participando de manera activa en ellas, y así lo reconoce la legalidad vigente. Y no sólo por el simple hecho de que nadie debe ser ajeno a la evolución y desarrollo del entorno en que habita, ya que directa o indirectamente le afectan las decisiones que se toman, sino por mera transparencia del proceso urbanístico. Además del interés general que para un sector de población cada vez más importante puede tener todo lo relacionado con un desarrollo más equilibrado, el tipo de ciudad deseado, la calidad del espacio público que habita. Sin embargo, éste sigue siendo uno de los mecanismos más complicados de optimizar, debido fundamentalmente a la falta de una coparticipación organizada bajo protocolos eficaces contrastados, y a la dificultad a la hora de jerarquizar convenientemente las aportaciones recibidas.

En base a la experiencia, éste es uno de los hitos críticos de todo el proceso, pues puede convertirse en origen de conflictos. Superada la fase de la interpretación global del resultado de las propuestas, es importante aparcar el recelo con el que tendemos a ver cualquier maniobra de la administración pública respecto de las modificaciones urbanísticas introducidas en los procesos ya aprobados y en curso. Sobre todo, por parte de quienes habitan recientemente dentro de los ámbitos afectados.

La particularidad del caso que nos ocupa, que actúa en un suelo urbano cuyas parcelas edificables han de transformarse respecto de su estado inicial conviviendo con otras ya edificadas, lo hace si cabe aún más vulnerable. Por lo tanto, cualquier modificación planteada en su ámbito de actuación afecta muy directamente y de forma particularmente sensible a sus habitantes. Y todavía en mayor grado en los casos de edificaciones ya materializadas. Por lo que es necesario tener en cuenta que las modificaciones que puedan proponerse sean fáciles de comprender. Con unos planos de ordenación sencillos en los que se reflejen los bloques edificables, indicando las alturas de la edificación o el número máximo de plantas. Incluso mediante modelos físicos. La exposición tridimensional del planeamiento vigente comparado con el de las diferentes alternativas de modificación, resulta por lo general suficiente para hacerse una idea cierta de la modificación urbanística propuesta. Comprobando los datos de aprovechamiento o edificabilidad, ocupación, calificaciones pormenorizadas, etc., en un cuadro comparativo con el planeamiento vigente para que el nivel de conocimiento sea completo. (Al final del presente artículo se aporta un ejemplo del caso de varios sectores ubicados tanto al Este como al Oeste de la ciudad de Vitoria-Gasteiz: Salburua y Zabalgana respectivamente, tal y como fueron explicados según estas pautas en la comunicación definitiva, y tal y como ha vuelto a ser explicado tras la aprobación de la segunda re-densificación operada recientemente en los mismos ámbitos).

Además, y con el objeto de acercar posturas en este sentido, es interesante trabajar juntamente con el Consejo Asesor de Planeamiento Municipal, y con cuantos entes sociales proponga el órgano correspondiente. Permitiendo durante el período de participación pública potenciar su capacidad de analizar e informar acerca de las propuestas que se estimen oportunas. De este modo, el Ayuntamiento, vistas las alegaciones, las sugerencias y los informes, resolverá adoptando las decisiones que procedan para su incorporación al documento definitivo. A partir de su redacción consensuada, comienza el trámite de aprobación. Primero inicial, abriendo el periodo de exposición pública con su publicación en el Boletín Oficial correspondiente, sus comunicados en la prensa, y las notificaciones a las administraciones públicas con competencias sectoriales.

ACE, X (XX) CC BY-ND 3.0 ES | UPC Barcelona, España | Otros procedimientos de intervención en la ciudad contemporánea: sobre la re-densificación. DOI: http://dx.doi.org/10.5821/ace.15.45.9527 
A la vista del resultado del proceso de información pública y tras estudiar, informar y pronunciarse sobre las alegaciones presentadas, el documento incorporará las modificaciones que procedan. Pudiéndose ya proceder a la aprobación provisional, y tras el informe favorable de la Comisión de Ordenación del Territorio pertinente, la aprobación definitiva.

\subsection{Fase III. Modificación de los Planes Parciales y redacción de los PEOU o PERU correspondientes si así fueran necesarios}

Sobre la base ya elaborada en la Fase I para la preparación de la revisión de planeamiento (siendo necesario recoger las modificaciones que en su caso procedan como consecuencia de los períodos legales de participación pública, de las decisiones municipales, y de los informes correspondientes de otros organismos competentes), se redactarán sucesivamente las Modificaciones de los Planes Parciales, con el objeto de localizar pormenorizadamente los incrementos de las edificabilidades y dotaciones correspondientes. Se trata del paso final hacia las figuras urbanísticas que materializan los datos desarrollados durante el proceso de re-densificación. Complementando a los anteriores documentos de planeamiento de acuerdo con los criterios acordados. Dado el carácter de mayor rango de desarrollo de la figura del Plan General, no debe iniciarse su tramitación en tanto éste no esté aprobado definitivamente. Sin embargo, parece lógico que, desde el momento de la aprobación inicial de la revisión del planeamiento general, puedan adelantarse las modificaciones de Planes Parciales, o la redacción de los Planes Especiales de Ordenación Urbana complementarios. Aunque conlleva un cierto riesgo, agiliza el siempre lento proceso de tramitación de figuras urbanísticas. Cuyo inicio casi siempre comienza con retraso; cuando ya necesitarían ser operativas.

Figura 1. Plano esquemático de la estructura de la ciudad de Vitoria-Gasteiz orientado al Norte, con los sectores de desarrollo hacia el este (Salburua) y el oeste (Zabalgana)

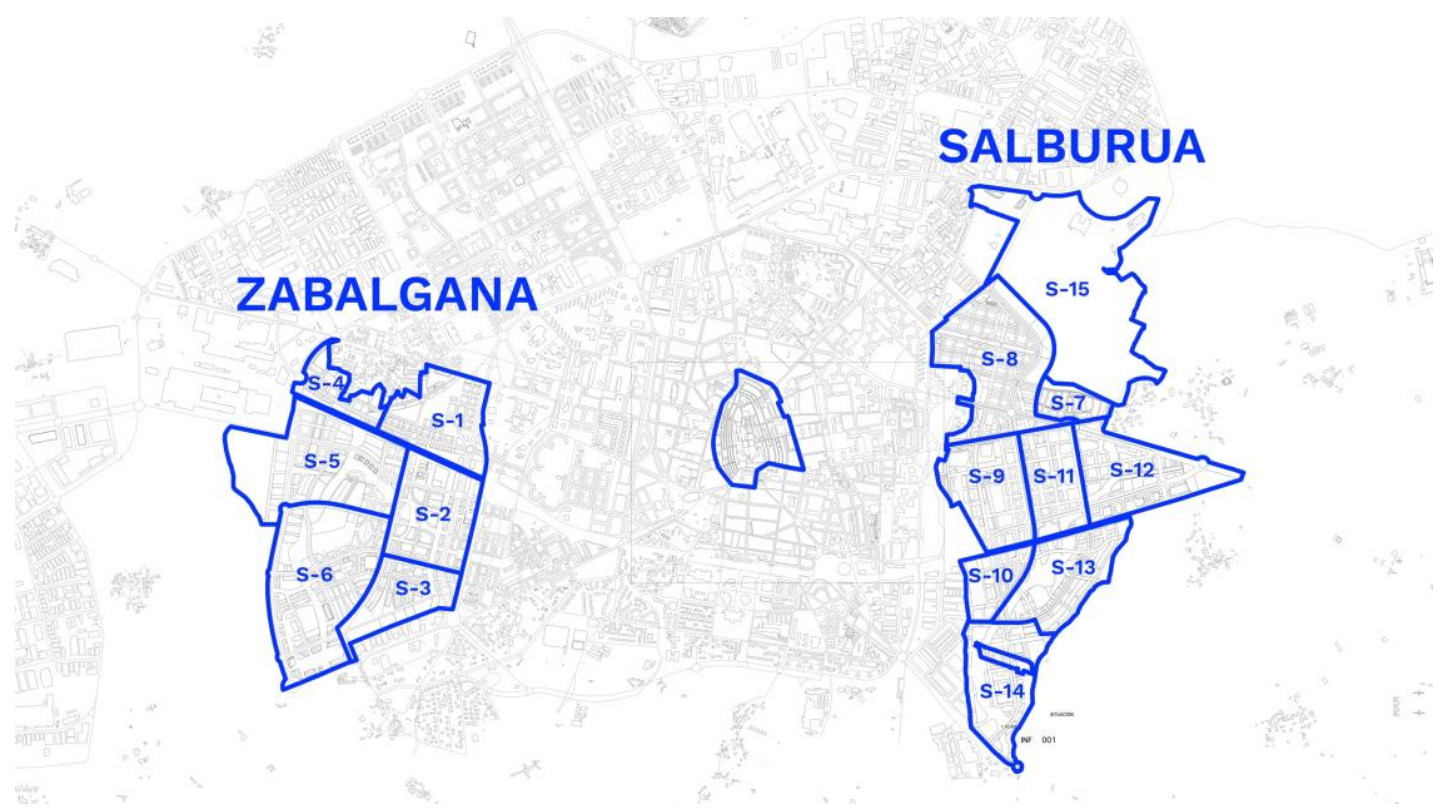

Fuente: Elaboración propia del autor del artículo.

Casi todos los sectores reflejados en el plano de situación anterior (Figura 1) han sufrido el proceso de re-densificación descrito, algunos de ellos hasta en dos ocasiones. Configurándose tras un proceso participativo que responde de forma general al esquema expuesto, tal y como se anuncia en los folletos explicativos definitivos.

ACE, X (XX) CC BY-ND 3.0 ES | UPC Barcelona, España | Otros procedimientos de intervención en la ciudad 14 contemporánea: sobre la re-densificación. DOI: http://dx.doi.org/10.5821/ace.15.45.9527 


\section{Gráficos comparativos del resultado final}

A continuación, se ofrecen una serie de gráficos (Figuras 2-7) que explican el resultado final del proceso en comparación con la situación inicial respecto de la primera re-densificación practicada en torno al año 2010. Nótese el código de colores que explica los incrementos practicados tanto sobre parcelas residenciales existentes como sobre otras nuevas. El código volumétrico a base de prismas repetitivos es simplemente indicativo de la posible máxima edificabilidad materializable.

Figuras 2 y 3. Recreación esquemática de la re-densificación de los sectores 1, 4 /

\section{2, 3, 5, 6 Zabalgana}

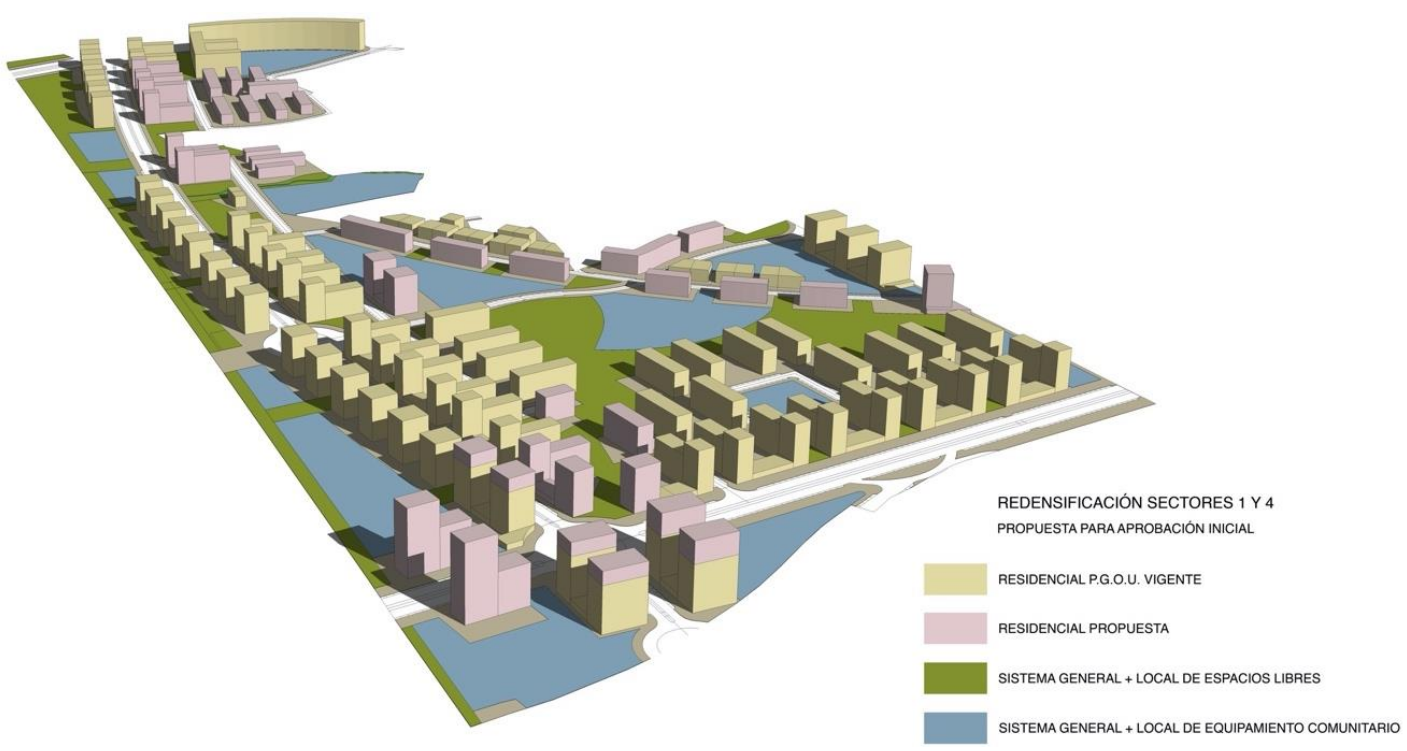

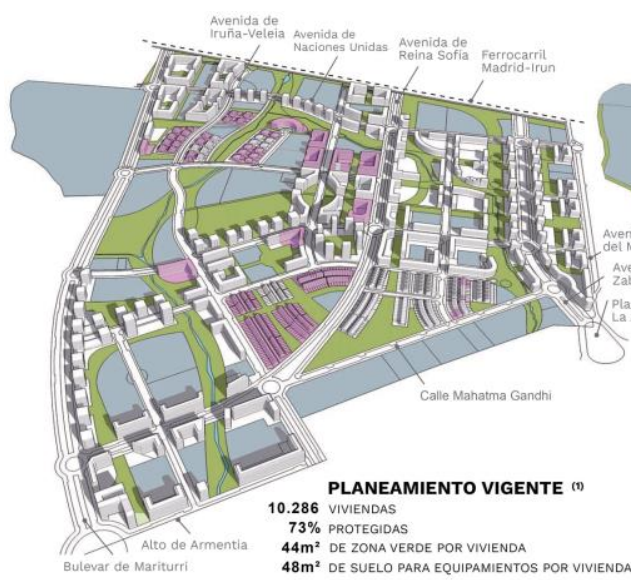

ZABALGANA [Sectores 2, 3, 5 y 6] Superficie total: $1.991 .735 \mathrm{~m}^{2}$

Zonas verdes

Equipamientos

Viviendas construidas o en proceso

Viviendas propuestas en la nueva alternativa

axis Altura de las viviendas previstas. $A=A$ tico, B=Bajo, E=Entrecubierta.

(1) Para la estimación del no de viviendas se han considerado viviendas equivalentes de $100 \mathrm{~m}^{2}$ construidos.

Fuente: Fragmento de los folletos divulgativos del Ayuntamiento, de Vitoria/Gasteiz 2010. Gráficos elaborados por el autor del artículo.

ACE, X (XX) CC BY-ND 3.0 ES | UPC Barcelona, España | Otros procedimientos de intervención en la ciudad contemporánea: sobre la re-densificación. DOI: http://dx.doi.org/10.5821/ace.15.45.9527 
Figuras 4 y 5. Recreación esquemática de la re-densificación de los sectores 7, 8 ,

$9,10,11 / 12,13,14$ Salburua
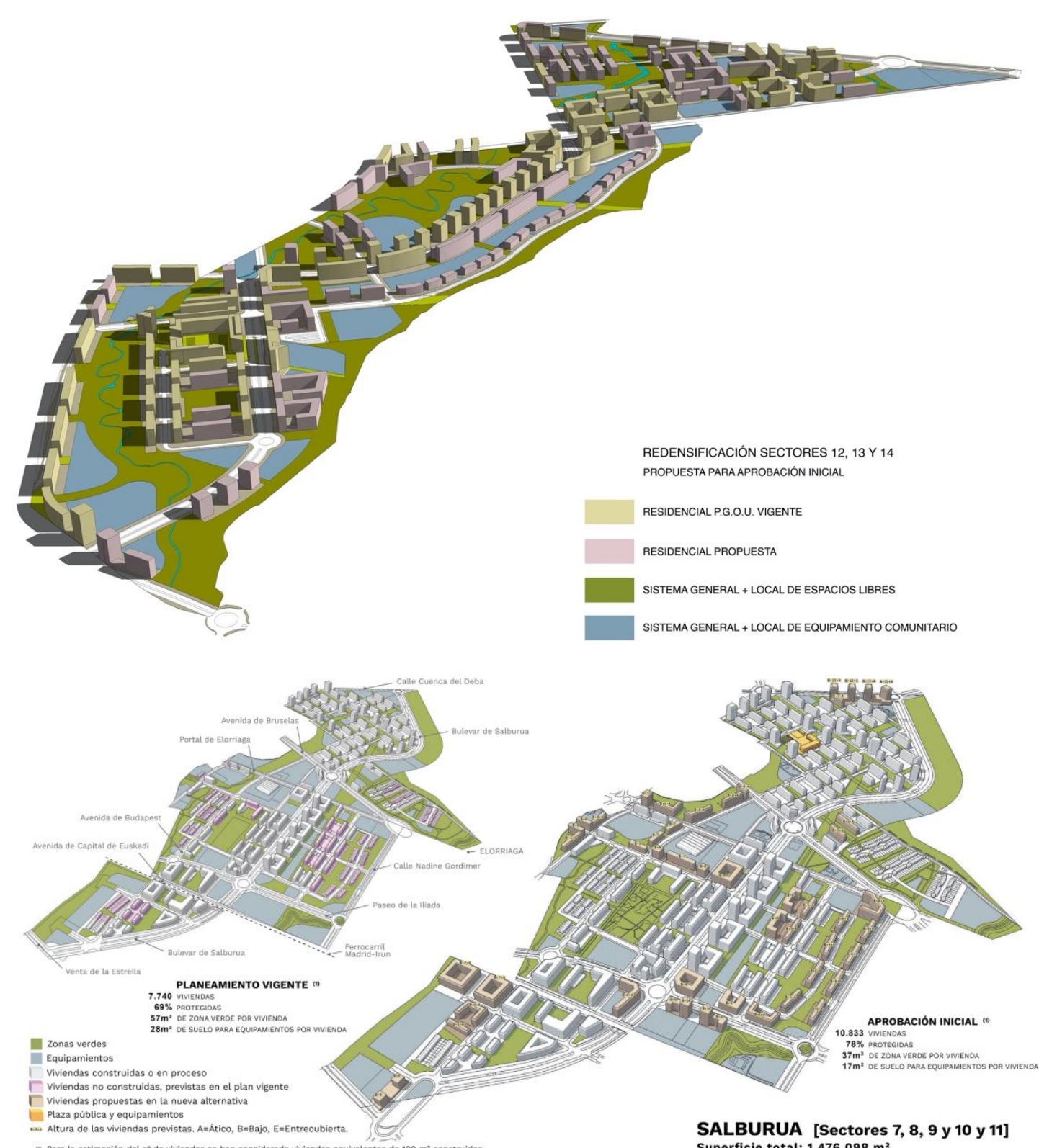

Fuente: Fragmento de los folletos divulgativos del Ayuntamiento, de Vitoria/Gasteiz 2010. Gráficos elaborados por el autor del artículo.

A continuación, se ofrece una segunda serie de gráficos que explican el resultado de la segunda redensificación practicada en el año 2020. Nótese que cambia el código de colores que explica los incrementos practicados, ya que en este caso se tienen en cuenta la naturaleza de las diferentes propiedades (administración y particulares). El código volumétrico también varía, y considera las diferentes tipologías de las edificaciones materializadas en parte o totalmente, así como aquéllas que ya disfrutan de licencia de obras.

ACE, X (XX) CC BY-ND 3.0 ES | UPC Barcelona, España | Otros procedimientos de intervención en la ciudad 16 contemporánea: sobre la re-densificación. DOI: http://dx.doi.org/10.5821/ace.15.45.9527 


\section{ACE Architecture, City and Environment}

E-ISSN 1886-4805

Figuras 6 y 7. Imágenes explicativas de la nueva propuesta de re-densificación
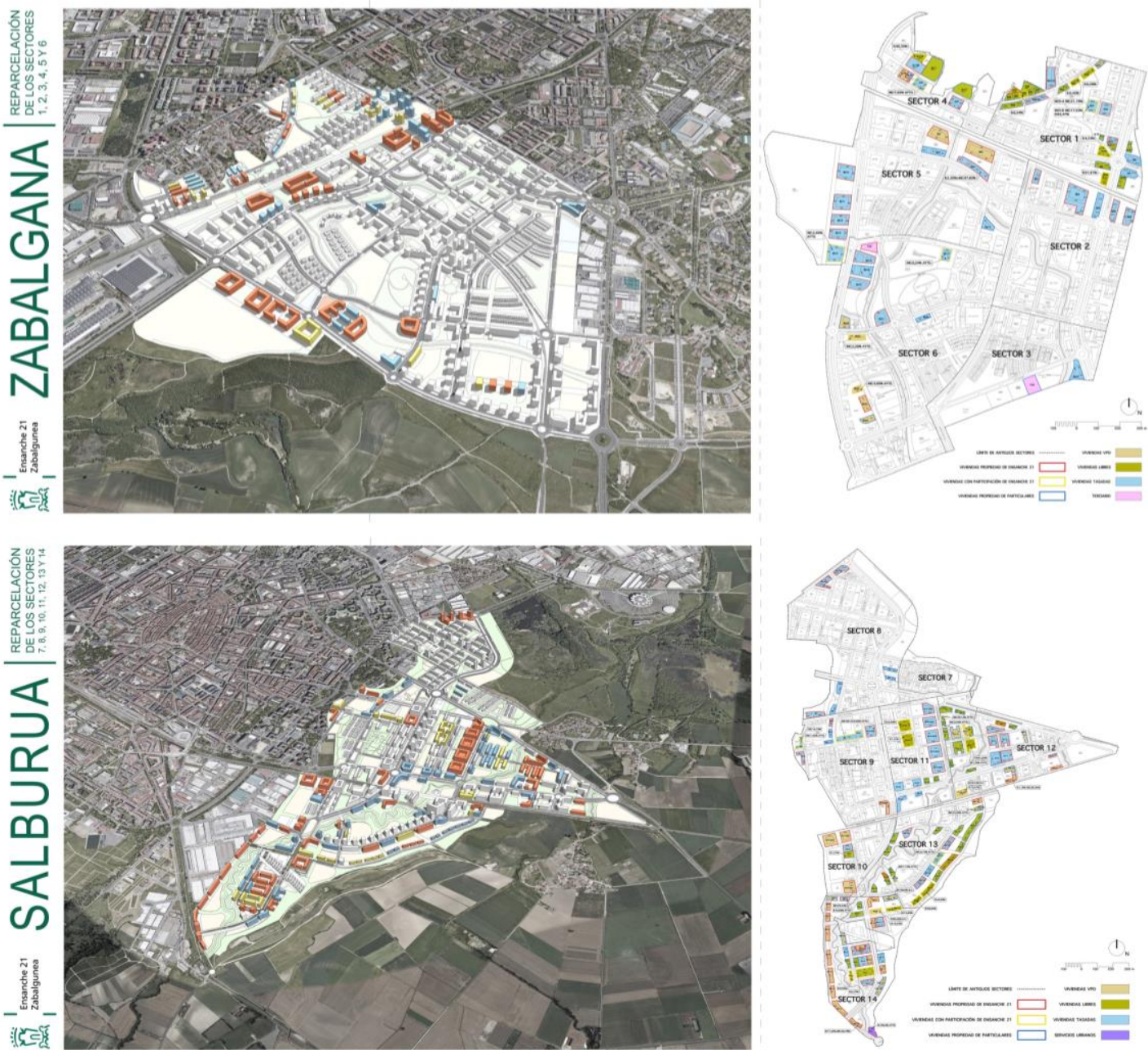

Fuente: Fragmento de los folletos divulgativos del Ayuntamiento, de Vitoria/Gasteiz 2020. Gráficos elaborados por el autor del artículo.

\section{Conclusiones}

La hipótesis inicial defendía que la re-densificación es una herramienta eficaz y novedosa a la hora de corregir intensidades de usos, discontinuidades, o incluso renovaciones urbanas puntuales de importancia. La descripción del proceso acaecido así lo demuestra en la práctica, ya que los nuevos desarrollos urbanos hacia el Este y Oeste de Vitoria-Gasteiz, hasta cierto punto han "corregido" un excesivo consumo de suelo.

Para apuntalar esta idea podemos formular una serie de preguntas acerca de su aplicación en los cascos urbanos consolidados, cuyas respuestas concretas, ya deslizadas de forma sucinta a lo largo del texto, operan como conclusiones.

- ¿Qué condiciones deben darse para que sea eficaz esta herramienta?

Se trata de una medida práctica ajustable a las necesidades reales, por lo que no demanda condiciones específicas de partida (más que la voluntad política y el consenso ciudadano).

ACE, X (XX) CC BY-ND 3.0 ES | UPC Barcelona, España | Otros procedimientos de intervención en la ciudad contemporánea: sobre la re-densificación. DOI: http://dx.doi.org/10.5821/ace.15.45.9527 
- ¿En qué medida puede corregir y complementar al urbanismo existente?

Se trata en realidad de un complemento de emergencia al planeamiento de rango general, o en su caso de la alternativa más eficaz a la redacción de un nuevo PGOU, ya que puede estructurarse como revisión parcial del mismo, base de las figuras urbanísticas de menor rango necesarias para su materialización.

- ¿Cuál es el ámbito idóneo para el uso de la re-densificación como herramienta de corrección urbanística?

Fundamentalmente lo constituyen los ámbitos urbanos hace ya tiempo consolidados, o en su caso, y como alternativa, los totalmente nuevos y en absoluto consolidados. Pero a ser posible nunca los semiconsolidados, fundamentalmente por dos razones: Por un lado, las quejas de los nuevos vecinos por el repentino y prematuro cambio de sus expectativas. Y por el otro, el ridículo que pesa sobre la administración competente por su falta de previsión a corto plazo.

- ¿Supone la re-densificación una nueva medida de actuación urbanística?

Sí hasta cierto punto puesto que supone el instrumento básico para crecer hacia adentro, y en el contexto actual muy sensible hacia temas relacionados con la sostenibilidad como el uso del suelo, este aspecto es de importancia capital. El problema es que dentro del modelo desarrollista en el que todavía estamos inmersos, si se opta por re-densificar, se hace principalmente en nuevos desarrollos todavía sin consolidar y rara vez en los ya existentes; creando elementos urbanos dispares y discontinuidades formales manifiestas.

- ¿En qué radica el interés de la re-densificación?

Sobre todo, en su grado adaptable de complejidad, que la convierte en una herramienta muy versátil como "medicina urbana"; poco o muy agresiva según las necesidades. Su espectro abarca desde llenar simples huecos o subir más alturas de las inicialmente permitidas, hasta modificar tramas y trasladar usos.

- ¿Podrían enumerarse algunas de sus ventajas?

- Restringe el uso de suelo, y por tanto el consumo de un bien no renovable.

- Renueva el tejido urbano, revitalizándolo.

- Lo adapta adecuadamente a necesidades nuevas y cambiantes.

- Permite una simultaneidad de actuaciones integradas.

- Es compatible con figuras urbanísticas de diferentes rangos.

- El nivel y riesgo de una posible equivocación es limitado.

- Opera siempre sobre un marco ya testado.

- Aprovecha buena parte de las infraestructuras y servicios sin mayor gasto.

- Otorga forma a la ciudad.

- Permite un abanico de intensidades.

- ¿Y alguno de sus inconvenientes?

- La oposición vecinal, fundamentalmente por falta de información.

- La necesidad de una visión global integrada desde la Administración competente.

ACE, X (XX) CC BY-ND 3.0 ES | UPC Barcelona, España | Otros procedimientos de intervención en la ciudad contemporánea: sobre la re-densificación. DOI: http://dx.doi.org/10.5821/ace.15.45.9527 


\section{Agradecimientos}

Me gustaría mostrar mi agradecimiento a las personas que han evaluado con rigor y participado con profesionalidad en el perfeccionamiento formal de este artículo; destacando su buen hacer y amplitud intelectual respecto de un trabajo que comparte una investigación situada en el difícil equilibrio entre la aproximación teórica y la experiencia práctica.

Conflicto de intereses: El autor declara que no hay conflicto de intereses.

\section{Bibliografía}

Arellano Ramos, B., \& Roca Cladera, J. (2012). Urban Sprawl en Europa: las periferias de las metrópolis, principal escenario de la dispersión de la urbanización. ACE: Architecture, City and Environment, 20, 95-114. https://doi.org/10.5821/ace.v7i20.2580

Bohigas, O. (2004). Contra la incontinencia urbana. Reconsideración moral de la arquitectura y la ciudad. Perspectivas Urbanas, 5, 52-54. https://upcommons.upc.edu/handle/2099/636

De Gregorio Hurtado, S. (2019). El enfoque integrado y la participación en la política urbana de la unión europea. El caso de la Iniciativa Urbana (2007-2013) en España. ACE: Architecture, City and Environment, 14(41), 35. https://doi.org/10.5821/ace.14.41.6763

Fariña Tojo, J. (2014). Necesidad de un cambio de modelo en la organización territorial. Primacía de lo local y el desarrollo endógeno como criterio básico de sostenibilidad. Palacio Miramar, Donostia-San Sebastián. En EESAP5 5 Congreso europeo sobre eficiencia energética y sostenibilidad en arquitectura y urbanismo. 7-9 de julio de 2014.

Gaja i Díaz, F. (2005). ¿Re-densificación de las ciudades Españolas? Los efectos urbanísticos de la especulación inmobiliaria. V Seminario Internacional de Suelo Urbano. La Re-densificación de la Ciudad Central a debate ¿Para qué, para quién, cómo? Universidad Nacional Autónoma de México (UNAM)-Programa Universitario de Estudios sobre la Ciudad (PUEC)-Lincoln Institute of Land Policy (LILP)". Ciudad de Mexico, Recuperado de http://personales.upv.es/fgaja/publicaciones/unam.pdf

Gehl, J. (2010). Cities for People. Washington DC, USA. Island Press.

Górgolas, P. (2018). El reto de compactar la periferia residencial contemporánea: densificación eficaz, centralidades selectivas y diversidad funcional. ACE Architecture City and Environment, 13(38), 57-80. https://doi.org/10.5821/ace.13.38.5211

Jacobs, J. (1961). The Death and Life of Great American Cities. Edición en español, Barcelona, Capitán Swing 2011.

López de Lucio, R. (1993). Ciudad y urbanismo a finales del siglo XX. Universidad de Valencia.

López de Munain, E. (2012). Procedimientos, agentes, actitudes, problemas y resultados de los programas de densificación (El caso de la redensificación de Vitoria). Territorios En Formación, O(2), 65-78. http://dx.doi.org/10.5821/ace.13.38.5211

Moix, LL (2010.). Arquitectura milagrosa. Barcelona, Anagrama.

ACE, X (XX) CC BY-ND 3.0 ES | UPC Barcelona, España | Otros procedimientos de intervención en la ciudad contemporánea: sobre la re-densificación. DOI: http://dx.doi.org/10.5821/ace.15.45.9527 
Penchyna, D. y Zedillo, C. (2018). Re-densificación Urbana. Arquine, Mexico.

Rincón Avellaneda, P. (2004). Análisis de los procesos de re-densificación en Bogotá. Bitácora Urbano $\begin{array}{lcc}\text { Territorial, } & 8(1) & \text { Recuperado } \\ \text { https://revistas.unal.edu.co/index.php/bitacora/article/view/18760 } & \end{array}$

Rowe, C. y Koetter, F. (1978). Ciudad Collage. Edición en español, Barcelona, GG reprints 1981-98.

Terán, F. (2009). Planeamiento urbano en la ciudad dispersa. Minerva, 4(12), 63-66. http://www.revistaminerva.com/articulo.php?id=355 